\title{
(6) OPEN ACCESS \\ Five-minute Apgar score as a marker for developmental vulnerability at 5 years of age
}

\author{
Neda Razaz, ${ }_{1}^{1,2}$ W Thomas Boyce, ${ }^{3}$ Marni Brownell, ${ }^{4}$ Douglas Jutte, ${ }^{5}$ Helen Tremlett, ${ }^{6}$ \\ Ruth Ann Marrie, ${ }^{7}$ K S Joseph ${ }^{1,2}$
}

- Additional material is published online only. To view please visit the journal online (http://dx.doi.org/10.1136/ archdischild-2015-308458).

For numbered affiliations see end of article.

\section{Correspondence to} Neda Razaz, Women's Hospital of British Columbia, Room C403-4500 Oak Street, Vancouver, British Columbia, Canada V6H 3N1; Neda.Razaz@gmail.com

Received 21 February 2015 Revised 2 June 2015 Accepted 11 June 2015 Published Online First 17 July 2015

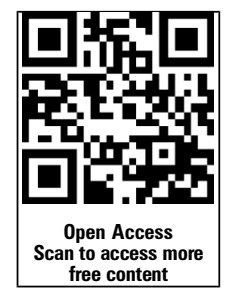

\section{SLinked}

- http://dx.doi.org/10.1136/ fetalneonatal-2015-308483

CrossMark

\footnotetext{
To cite: Razaz N, Boyce WT, Brownell M et al. Arch Dis Child Fetal Neonatal Ed 2016;101: F114-F120.
}

\section{ABSTRACT \\ Objective To assess the relationship between the $5 \mathrm{~min}$ Apgar score and developmental vulnerability at 5 years of age. \\ Design Population-based retrospective cohort study. \\ Setting Manitoba, Canada. \\ Participants All children born between 1999 and 2006 at term gestation, with a documented 5 min Apgar score. \\ Exposure 5 min Apgar score. \\ Main outcome measures Childhood development at 5 years of age, expressed as vulnerability (absent vs \\ What is already known on this topic \\ Low 5 min Apgar scores are associated with adverse short-term and long-term cognitive outcomes and developmental impairment. \\ - A comprehensive view of the relation between Apgar scores and the child's early developmental health has been largely overlooked in the existing literature.} present) on five domains of the Early Development Instrument: physical health, social competence, emotional maturity, language and cognitive development, and communication skills.

Results Of the 33883 children in the study, most (82\%) had an Apgar score of 9; $1 \%$ of children had a score $<7$ and $5.6 \%$ had a score of 10 . Children with Apgar scores $<10$ had higher odds of vulnerability on the physical domain at age 5 years compared with children with a score of 10 (eg, adjusted OR (aOR) for Apgar $9=1.23,95 \% \mathrm{Cl}$ 1.05 to 1.44). Similarly, children with Apgar scores of $<10$ were more vulnerable on the emotional domain (eg, aOR for Apgar $9=1.20,95 \% \mathrm{Cl} 1.03$ to 1.41). Nevertheless, the Apgar-based prognostic model had a poor sensitivity for physical vulnerability $(19 \%, 95 \% \mathrm{Cl} 18 \%$ to $20 \%)$. Although the Apgar score-based prognostic model had reasonable calibration ability and risk-stratification accuracy for identifying developmentally vulnerable children,

classification accuracy was poor.

Conclusions The risk of developmental vulnerability at 5 years of age is inversely associated with the $5 \mathrm{~min}$ Apgar score across its entire range, and the score can serve as a population-level indicator of developmental risk.

\section{INTRODUCTION}

Introduced by Virginia Apgar in 1952, the Apgar score measures the health status of newborn children based on heart rate, respiration, colour, muscle tone and reflex irritability. ${ }^{1}$ The Apgar score at birth and $1 \mathrm{~min}$ after birth was initially developed to assess the immediate condition of the newborn and the potential need for resuscitation. Subsequent studies have demonstrated the $5 \mathrm{~min}$ Apgar score's ability to predict longer-term cognitive outcomes such as a reduced IQ, a lower numeracy and literacy score, ${ }^{2}$ neurological disability and low cognitive function at age 19 years. ${ }^{3}$ In school-age children, low Apgar scores have been linked to minor motor, language, speech and developmental impairments. ${ }^{4-6}$

Most follow-up studies examining the long-term prognostic value of the Apgar score on child

\section{What this study adds}

The 5 min Apgar score is inversely associated with a risk of developmental vulnerability in early childhood across its entire range.

- There is an increased risk of developmental vulnerability among children with an Apgar score of 9, compared with children with a score of 10.

- The Apgar score based prognostic model created reasonably acceptable categories of low and high-risk children for developing an early intervention program.

outcomes have focused on the association between extremely low 5 min Apgar scores, such as those linked to severe birth asphyxia, and distinct neurocognitive profiles such as IQ and academic ability. Virtually, no prior study has examined the developmental correlates of the Apgar score as a continuous variable, across the entire spectrum of recorded scores. ${ }^{2} 78$ In addition, there are no studies that have assessed the prognostic performance of the 5 min Apgar score for identifying developmental vulnerability among school-age children. A programme of early diagnosis could identify high-risk children who would potentially benefit from interventions designed to mitigate the developmental problems at school-going age. We, therefore, carried out a population-based study to comprehensively examine the relationship between the 5 min Apgar score and developmental vulnerability at 5 years of age.

\section{METHODS}

Study design

All children born in Manitoba, Canada, between 1 January 1999 and 31 December 2006, with a gestational age of 37 weeks or greater and a 
documented 5 min Apgar score, as well as a completed Early Development Instrument (EDI) assessment at age 5 years between 2005 and 2011, were included in the study cohort. Data were obtained from the Manitoba Population Health Research Data Repository, which collates information on health and social services utilisation for all residents of Manitoba. ${ }^{9}$ Two data sources were used to determine the socioeconomic status (SES) of children in the study: the provincial Employment and Income Assistance data (identifying those requiring social assistance or income supplementation) and Census data (providing mean household income by area of residence). Finally, $\mathrm{EDI}^{10}$ data, which provided information on early childhood developmental outcomes and school readiness, were accessed through linkage with the Healthy Child Manitoba Office. All data files used in this study were anonymised, and linkage at the individual level was performed using a scrambled personal health identification number. The reliability and validity of these data sources have been previously documented. ${ }^{11} 12$

\section{Prognostic variables and outcomes}

The primary prognostic variable of interest was the routinely collected 5 min Apgar score (hereafter referred to as the Apgar score') from the hospital record. Apgar scores were analysed in several ways: (a) using conventional categories: Apgar values of $0-6,7,8$ and 9-10; (b) revised categories: Apgar values of $0-3$, $4,5,6,7,8,9$ and 10 (values from 0 to 3 were grouped together because of small numbers); (c) as a continuous variable and (d) as a continuous variable with a quadratic term to evaluate the potential for a non-linear relationship with developmental outcomes.

Other independent variables included in the prognostic model were infant sex (male vs female), birth weight-forgestational age, age of the child in years at the time of EDI assessment, gestational age at birth in completed weeks $(37,38$, $39,40,41$ and $\geq 42$ ), breastfeeding initiation (yes vs no) and SES. Birth weight-for-gestational age was categorised as: small $(<10$ th percentile), appropriate (10th-90th percentile) and large (>90th percentile). ${ }^{13}$ Each child's family income was derived from the mean household income in the child's residential area (based on postal code) obtained from the 2006 Canadian Census data. ${ }^{14-16}$ This was complemented by parental receipt of income assistance at any time from the child's birth to the EDI assessment. Family SES was based on income quintile and receipt of income assistance, and was categorised as low (quintile 1 or income assistance recipients) ${ }^{12}$ medium (quintiles 2 and 3 ) and high (quintiles 4 and 5).

The primary outcome of interest was childhood development, as measured by the EDI. The EDI was routinely administered biennially in all 37 public school divisions in Manitoba beginning in 2005/06. Teachers-remunerated by their school district-completed the EDI for each child in their kindergarten class (age range 5-7 years) midway through the school year. The EDI consists of 104 binary and Likert-scale items designed to tap five core areas of early childhood development: ${ }^{10} 17$ physical health and well-being; social competence, emotional maturity, language and cognitive development and communication skills and general knowledge. Children were considered vulnerable on a domain if their scores fell below the 10 th percentile value ${ }^{18}$ based on the national EDI scores. ${ }^{19}$

\section{Statistical analyses}

The frequency of each Apgar-score value was calculated within categories of maternal and infant characteristics. Variables chosen as prognostic determinants were selected because of presumed association with developmental vulnerability based on the literature, ${ }^{18} 20$ as well as availability in our data source. Multivariable logistic regression was used to examine the association between each independent variable and vulnerability on each domain of the EDI. Results were expressed as ORs with 95\% CIs. Models were evaluated for goodness of fit using the Akaike information criterion (AIC).

In addition to modelling vulnerability on the EDI, we also assessed the performance of the prognostic model for identifying any developmental vulnerability. The model-predicted probability of vulnerability on each EDI domain was categorised as identifying low risk $(<10 \%)$, medium risk $(10 \%-19 \%)$ and high risk $(\geq 20 \%)$ of an adverse developmental outcome. The modelpredicted probability of vulnerability on any of the EDI domains was categorised as identifying low risk $(<30 \%)$, medium risk $(30 \%-44 \%)$ and high risk $(\geq 45 \%)$ of any adverse developmental outcome. These cut-off values were chosen based on the provincial prevalence of vulnerability, since approximately $11 \%$ of children are vulnerable on an EDI domain, and $28 \%$ of children are vulnerable on one or more domains. ${ }^{21}$ We assessed performance of the prognostic model in terms of calibration ability, stratification capacity and classification accuracy ${ }^{22} 23$ to identify children at high risk for developmental vulnerability.

A two-sided $\mathrm{p}$ value $<0.05$ was used to define statistical significance. No adjustment was made for multiple comparisons in this exploratory study. The University of Manitoba Health Research Ethics Board sanctioned the study, and the Manitoba Health Information Privacy Committee approved data access. Analyses were performed using SAS V.9.2 (SAS Institute, Cary, North Carolina, USA).

\section{RESULTS}

There were 33883 children (mean age $=5.7$ years) with a gestational age of $\geq 37$ weeks and complete Apgar and EDI data who were included in the study. The majority (82\%) of children had an Apgar score of 9, and $1 \%$ had a score $<7$. Only $0.2 \%$ of children had an Apgar score $\leq 4$ (table 1). As expected, low Apgar scores were also more common among males, small-for-gestational age live births, children of mothers who did not initiate breast feeding and those with a low SES.

The prevalence of vulnerability within each of the five EDI domains by Apgar score is shown in figure 1. Overall, the prevalence of vulnerability in one or more domains of the EDI was $28 \%$, with physical and language domains having the highest rates of vulnerability at $12 \%$ and $12 \%$, respectively. There was a graded, decreasing trend in the rate of vulnerability on all domains of the EDI with increasing Apgar score. Vulnerability rates for the physical (23\%), social (18\%) and emotional (16\%) domains were highest among children with an Apgar score of 5 .

The prognostic model for categorising children into low $(<10 \%)$, medium $(10 \%-19 \%)$ and high risk groupings $(\geq 20 \%)$ for vulnerability on the physical domain showed reasonable calibration ability; $7.6 \%$ and $25 \%$ of low-risk versus high-risk children were found to be vulnerable on the physical domain (table 2). Similar results were obtained with regard to calibration ability on the other EDI domains. The model also successfully stratified children into reasonably sized risk categories, with a large proportion of children classified as low or medium risk, and approximately $5 \%-12 \%$ of children identified as being at high risk for vulnerability in each of the five EDI domains. However, the classification accuracy of the model was poor; the model had a detection rate 


\section{Original article}

Table 1 Maternal and infant characteristics in the study population by Apgar score at $5 \mathrm{~min}$, Manitoba

\begin{tabular}{|c|c|c|c|c|c|c|c|c|c|c|c|c|c|c|c|c|}
\hline \multirow{2}{*}{$\begin{array}{l}\text { Birth and } \\
\text { demographic } \\
\text { characteristics }\end{array}$} & \multicolumn{2}{|l|}{ Total } & \multicolumn{2}{|c|}{ Apgar 0-4 } & \multicolumn{2}{|c|}{ Apgar 5} & \multicolumn{2}{|c|}{ Apgar 6} & \multicolumn{2}{|c|}{ Apgar 7} & \multicolumn{2}{|c|}{ Apgar 8} & \multicolumn{2}{|c|}{ Apgar 9} & \multicolumn{2}{|c|}{ Apgar 10} \\
\hline & No. & $\%$ & No. & $\%$ & No. & $\%$ & No. & $\%$ & No. & $\%$ & No. & $\%$ & No. & $\%$ & No. & $\%$ \\
\hline \multicolumn{17}{|l|}{ Sex of the child } \\
\hline Female & 16719 & 49 & 24 & 0.1 & 36 & 0.2 & 84 & 0.5 & 283 & 1.7 & 1556 & 9.31 & 13776 & 82 & 960 & 5.7 \\
\hline Male & 17164 & 51 & 36 & 0.2 & 45 & 0.3 & 103 & 0.6 & 326 & 1.9 & 1865 & 10.9 & 13850 & 81 & 939 & 5.5 \\
\hline \multicolumn{17}{|c|}{ Child's age at EDI completion (years) } \\
\hline Mean (SD) & 33883 & 0.5 & 60 & $5.7(0.3)$ & 81 & $5.6(0.4)$ & 187 & $5.6(0.4)$ & 609 & $5.7(0.4)$ & 3421 & $5.7(0.3)$ & 27605 & $5.7(0.7)$ & 1899 & $5.7(0.7)$ \\
\hline \multicolumn{17}{|c|}{$\begin{array}{l}\text { Neighbourhood income } \\
\text { (SES) }\end{array}$} \\
\hline Highest SES & 17215 & 51 & 26 & 0.2 & 38 & 0.2 & 85 & 0.5 & 316 & 1.8 & 1666 & 9.7 & 14174 & 82 & 910 & 5.3 \\
\hline Middle SES & 12328 & 36 & 25 & 0.2 & 30 & 0.2 & 69 & 0.6 & 226 & 1.8 & 1330 & 11 & 9873 & 80 & 775 & 6.3 \\
\hline $\begin{array}{l}\text { Lowest SES or } \\
\text { income assistance }\end{array}$ & 4326 & 13 & 8 & 0.2 & 13 & 0.3 & 33 & 0.8 & 67 & 1.5 & 422 & 9.8 & 3570 & 83 & 213 & 4.9 \\
\hline \multicolumn{17}{|l|}{ Gestational weeks } \\
\hline 37 & 2253 & 6.7 & $<5$ & $<0.1$ & $<5$ & $<0.1$ & 12 & 0.5 & 59 & 2.6 & 301 & 13 & 1803 & 80 & 78 & 3.5 \\
\hline 38 & 4951 & 15 & 10 & 0.2 & 11 & 0.2 & 25 & 0.5 & 74 & 1.5 & 551 & 11 & 4008 & 81 & 272 & 5.5 \\
\hline 39 & 7901 & 23 & 15 & 0.2 & 18 & 0.2 & 34 & 0.4 & 113 & 1.4 & 660 & 8.4 & 6638 & 84 & 423 & 5.4 \\
\hline 40 & 13205 & 39 & 20 & 0.2 & 30 & 0.2 & 74 & 0.6 & 250 & 1.9 & 1277 & 9.7 & 10760 & 82 & 794 & 6.0 \\
\hline 41 & 5167 & 15 & 12 & 0.2 & 13 & 0.3 & 38 & 0.7 & 104 & 2.0 & 581 & 11 & 4106 & 80 & 313 & 6.1 \\
\hline$\geq 42$ & 396 & 1.2 & $<5$ & $<0.1$ & 6 & 1.5 & $<5$ & $<0.1$ & 9 & 2.3 & 51 & 13 & 311 & 79 & 19 & 4.8 \\
\hline \multicolumn{17}{|c|}{ Birth weight for gestational age } \\
\hline Appropriate & 26447 & 78 & 41 & 0.2 & 66 & 0.3 & 131 & 0.5 & 456 & 1.7 & 2582 & 9.8 & 21659 & 82 & 1512 & 5.7 \\
\hline Small & 2565 & 7.6 & 9 & 0.4 & 8 & 0.3 & 31 & 1.2 & 69 & 2.7 & 319 & 12 & 2026 & 79 & 103 & 4.0 \\
\hline Large & 4871 & 14 & 10 & 0.2 & 7 & 0.1 & 25 & 0.5 & 84 & 1.7 & 520 & 11 & 3941 & 81 & 284 & 5.8 \\
\hline \multicolumn{17}{|l|}{ Breastfeeding initiation } \\
\hline Yes & 28337 & 84 & 49 & 0.2 & 58 & 0.2 & 150 & 0.5 & 511 & 1.8 & 2871 & 10 & 23183 & 82 & 1515 & 5.3 \\
\hline No & 5546 & 16 & 11 & 0.2 & 23 & 0.4 & 37 & 0.7 & 98 & 1.8 & 550 & 9.9 & 4443 & 80 & 384 & 6.9 \\
\hline Total & 33883 & & 60 & 0.2 & 81 & 0.2 & 187 & 0.6 & 609 & 1.8 & 3421 & 10 & 27626 & 82 & 1899 & 5.6 \\
\hline
\end{tabular}

(sensitivity) between $11 \%$ and $26 \%$ for vulnerability on the five different domains of the EDI. Similar results were obtained for the prognostic model identifying vulnerability on any of the EDI domains (table 2).
Using the conventional categorisation of Apgar scores (table 3), we observed that compared with those with an Apgar score of 9-10, the odds of vulnerability on the physical domain of the EDI were significantly higher among children with an Apgar score of

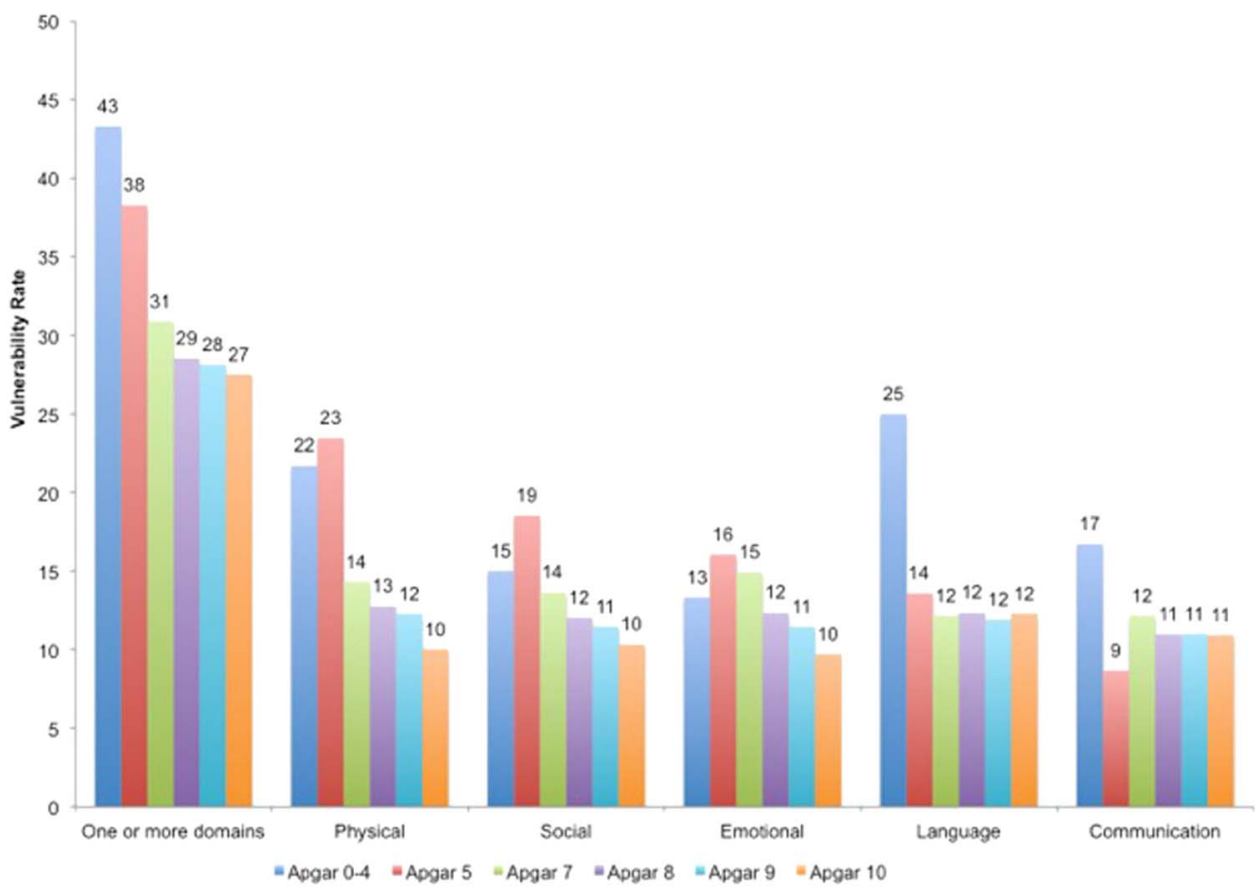

Figure 1 Rates of vulnerability within the five Early Development Instrument (EDI) domains by Apgar score at 5 min, Manitoba. Numbers for children with an Apgar score of $0-4$ were grouped because of small numbers. 
Table 2 Prognostic performance of the 5 min Apgar score-based model for identifying children with developmental vulnerability on each or any of the five Early Developmental Instrument (EDI) domains

\begin{tabular}{|c|c|c|c|c|c|c|c|}
\hline \multirow[b]{2}{*}{ EDI domains } & \multicolumn{2}{|l|}{ Calibration ability* } & \multicolumn{3}{|c|}{ Stratification capacity* } & \multicolumn{2}{|c|}{ Classification accuracy* } \\
\hline & $\begin{array}{l}\text { Probability of } \\
\text { vulnerability if in } \\
\text { high-risk group, } \%\end{array}$ & $\begin{array}{l}\text { Probability of } \\
\text { vulnerability if in } \\
\text { low-risk group, \% }\end{array}$ & $\begin{array}{l}\text { Low-risk } \\
\text { categoryt, \% }\end{array}$ & $\begin{array}{l}\text { Mid-risk } \\
\text { categoryt, \% }\end{array}$ & $\begin{array}{l}\text { High-risk } \\
\text { categoryt, \% }\end{array}$ & $\begin{array}{l}\text { Detection rate/ } \\
\text { sensitivity, \% } \\
(95 \% \mathrm{Cl})\end{array}$ & $\begin{array}{l}\text { False-positive } \\
\text { rate, } \%(95 \% \mathrm{Cl})\end{array}$ \\
\hline Physical & 25 & 7.6 & 39 & 52 & 9.4 & 19 (18 to 20$)$ & 8 (7 to 8$)$ \\
\hline Social & 24 & 6.0 & 43 & 48 & 9.7 & 20 (19 to 22$)$ & 8 (8 to 9$)$ \\
\hline Emotional & 23 & 6.0 & 48 & 44 & 7.8 & 16 (14 to 17$)$ & 7 (6 to 7$)$ \\
\hline $\begin{array}{l}\text { Language and } \\
\text { cognitive }\end{array}$ & 26 & 6.8 & 49 & 39 & 12 & 26 (24 to 27$)$ & $10(9$ to 10$)$ \\
\hline Communication & 24 & 7.3 & 54 & 41 & 5.2 & 11 (10 to 12$)$ & $4(4$ to 5$)$ \\
\hline Any EDI domain* & 51 & 21 & 60 & 31 & 9.3 & 17 (16 to 18$)$ & $6(6$ to 7$)$ \\
\hline
\end{tabular}

0-6 (OR 1.55, 95\% CI 1.17 to 2.06). A graded excess in odds of vulnerability was evident with increasing Apgar score though rates of developmental vulnerability among those with Apgar scores of 7 and 8 were not significantly different from those with an Apgar score of 9-10.

Analyses with modelling Apgar scores using the revised categories, however, revealed that the odds of vulnerability on the physical domain increased in a graded fashion across the full range of scores (table 4). Children with an Apgar score of 7, 8 or 9 had significantly higher odds of vulnerability on the physical domain compared with children with an Apgar score of 10 (OR 1.45, 1.24 and 1.23 for Apgar 7, 8 and 9, respectively; table 4). Similarly, children with an Apgar score of 7, 8 or 9 had higher odds of vulnerability on the emotional domain compared with children with an Apgar score of 10 (OR 1.60, 1.24 and 1.20 for Apgar 7, 8 and 9, respectively). Children with scores $<4$ had 2.71 times higher odds of vulnerability in the language domain compared with children with an Apgar score of 10 (OR $2.71,95 \%$ CI 1.22 to 6.01 ).

Finally, analyses with Apgar score modelled as a continuous variable (see online supplementary appendix table 1) showed that unit increase in the Apgar score was associated with a significant decrease in vulnerability on the physical (OR 0.91, 95\% CI 0.86 to 0.95 ), social (OR $0.94,95 \%$ CI 0.89 to 0.99 ) and emotional domains (OR 0.93, 95\% CI 0.89 to 0.98). Non-significant decreases in vulnerability were observed on the language and communication domain. Modelling Apgar scores as a continuous variable or as a continuous variable with a quadratic term did not improve model fit (as assessed by the AIC) or change the pattern of the findings.

\section{DISCUSSION}

Our population-based study showed an association between the Apgar score and early childhood developmental vulnerability at 5 years of age, with lower 5 min Apgar scores being associated, in a graded continuous manner, with an increased risk of vulnerability on the physical health and well-being, emotional and social domains of the EDI. The Apgar scorebased prognostic model created reasonably acceptable categories of low-risk and high-risk children for creating an early intervention programme, but failed to identify a significant proportion of children who were subsequently found to be vulnerable on one or more developmental domains. On the other hand, our study showed an increased risk of vulnerability on the physical and emotional domains even among children with an Apgar score of 9, compared with children with a score of 10 .

Although the literature suggests that low Apgar scores are of concern in the short and long term, our results showed that the negative association between Apgar scores and developmental adversity extended in a linear, graded fashion across the entire range of scores. Such a finding-revealing a continuous linear association with a conventionally categorical predictor variable - parallels the identification of SES as a graded, continuous, population-level predictor of morbidity, whereas previously only outright poverty was viewed as important for health and wellbeing. ${ }^{24}$ Our findings are consistent with previous studies showing an increased need for special education among children with Apgar scores of 7 or 8 at 5 min after birth compared with children with Apgar score 9 or 10 and the need for extra resources in kindergarten. ${ }^{2} 4820$

A neurobiological account for why Apgar scores might be significant, continuous-level predictors of developmental outcomes cannot be provided by the data available in this study. Many of the observable physiological components of the Apgar score are mediated by shifts in autonomic nervous system (ANS) activation, and autonomic reactivity to emotional and physical stressors (stressors such as labour and birth) has been linked to chronic disease processes in adults ${ }^{25}$ and to both developmental ${ }^{26}$ and health outcomes ${ }^{27}$ in children. Mothers' prenatal adversity, moreover, has been associated with trajectories of postnatal development of autonomic reactivity, ${ }^{28}$ suggesting that even emotional stressors antecedent to birth can influence ANS responses. Thus, the present finding-that the full range of Apgar scores predicts developmental vulnerability at kindergarten-could represent the influence of heightened stress reactivity (indexed via ANS responses to the stress of birth) on long-term developmental endpoints.

Our prognostic model's findings are in line with previous studies that showed that the specificity of the Apgar score as a prognostic tool was far better than its sensitivity. ${ }^{329}{ }^{30}$ Further, risk assessment research also shows that biological criteria alone fail to identify $65 \%$ of children who, subsequently, experience high rates of poor health and disappointing educational outcomes. ${ }^{31}$ Although our findings indicate that Apgar scores cannot be effectively used at the individual level to determine prognosis or trigger preventive intervention, our findings could have 
Table 3 Adjusted ORs and $95 \%$ Cls showing the association between 5 min Apgar score (conventional categories) and vulnerability within the five EDI domains

\begin{tabular}{|c|c|c|c|c|c|c|c|c|c|}
\hline \multirow[b]{2}{*}{ Factors } & \multicolumn{3}{|c|}{$\begin{array}{l}\text { Physical health and well-being } \\
\text { domain }\end{array}$} & \multicolumn{3}{|c|}{ Social domain } & \multicolumn{3}{|c|}{ Emotional domain } \\
\hline & OR & $95 \% \mathrm{Cl}$ & $\mathrm{p}$ Value & OR & $95 \% \mathrm{Cl}$ & $\mathrm{p}$ Value & OR & $95 \% \mathrm{Cl}$ & $\mathrm{p}$ Value \\
\hline \multicolumn{10}{|c|}{ Apgar score at $5 \min$ (vs 5 min Apgar 9-10) } \\
\hline $0-6$ & 1.55 & (1.17 to 2.06$)$ & $<0.001$ & 1.36 & (1.01 to 1.84$)$ & 0.05 & 1.09 & (0.78 to 1.51$)$ & 0.62 \\
\hline 7 & 1.19 & (0.94 to 1.51$)$ & 0.14 & 1.21 & (0.95 to 1.54$)$ & 0.12 & 1.34 & (1.07 to 1.69$)$ & 0.01 \\
\hline 8 & 1.02 & (0.92 to 1.14 ) & 0.72 & 1.01 & (0.91 to 1.13$)$ & 0.84 & 1.04 & (0.94 to 1.17$)$ & 0.44 \\
\hline Sex of the child, male (vs female) & 1.75 & (1.63 to 1.87$)$ & $<0.0001$ & 2.51 & (2.33 to 2.70 ) & $<0.0001$ & 3.12 & (2.89 to 3.36 ) & $<0.0001$ \\
\hline Child's age at EDI completion (years) & 0.85 & (0.79 to 0.91$)$ & $<0.0001$ & 0.82 & (0.76 to 0.88 ) & $<0.0001$ & 0.86 & (0.80 to 0.92$)$ & $<0.0001$ \\
\hline \multicolumn{10}{|l|}{ SES (vs highest SES) } \\
\hline Middle SES & 2.40 & (2.19 to 2.63 ) & $<0.0001$ & 2.27 & (2.07 to 2.50$)$ & $<0.0001$ & 1.77 & (1.60 to 1.95$)$ & $<0.0001$ \\
\hline Lowest SES or income assistance & 1.47 & (1.36 to 1.58$)$ & $<0.0001$ & 1.44 & (1.33 to 1.55$)$ & $<0.0001$ & 1.19 & (1.11 to 1.29 ) & $<0.0001$ \\
\hline \multicolumn{10}{|l|}{ Gestational weeks (vs 40 weeks) } \\
\hline 37 & 1.24 & (1.09 to 1.42$)$ & $<0.01$ & 1.16 & (1.02 to 1.34$)$ & 0.03 & 1.13 & (0.98 to 1.29$)$ & 0.10 \\
\hline 38 & 1.07 & (0.97 to 1.19$)$ & 0.17 & 1.14 & (1.03 to 1.26$)$ & 0.01 & 1.06 & (0.95 to 1.18 ) & 0.28 \\
\hline 39 & 1.05 & (0.96 to 1.15$)$ & 0.28 & 1.09 & (0.99 to 1.19 ) & 0.06 & 1.06 & (0.97 to 1.16$)$ & 0.19 \\
\hline 41 & 1.01 & (0.92 to 1.12$)$ & 0.83 & 1.07 & (0.97 to 1.19 ) & 0.19 & 1.08 & (0.98 to 1.20$)$ & 0.12 \\
\hline$\geq 42$ & 1.24 & (0.93 to 1.65$)$ & 0.14 & 1.06 & (0.78 to 1.45$)$ & 0.71 & 1.44 & (1.09 to 1.90$)$ & 0.01 \\
\hline \multicolumn{10}{|c|}{ Birth weight for gestational age (vs 'appropriate') } \\
\hline Large & 1.09 & (0.99 to 1.19 ) & 0.09 & 1.02 & (0.93 to 1.13$)$ & 0.68 & 1.03 & (0.93 to 1.13$)$ & 0.59 \\
\hline Small & 1.33 & (1.19 to 1.49$)$ & $<0.0001$ & 1.26 & (1.12 to 1.42$)$ & $<0.001$ & 1.16 & (1.02 to 1.31$)$ & 0.02 \\
\hline \multirow[t]{2}{*}{ Breastfeeding initiation, yes (vs no) } & 1.41 & (1.30 to 1.53$)$ & $<0.0001$ & 1.40 & (1.29 to 1.52$)$ & $<0.0001$ & 1.16 & (1.06 to 1.27$)$ & 0.001 \\
\hline & & \multicolumn{4}{|c|}{ Language domain } & \multicolumn{4}{|c|}{ Communication domain } \\
\hline Factors & & $\mathrm{OR}$ & $95 \% \mathrm{Cl}$ & & $\mathrm{p}$ Value & OR & & & $\mathrm{p}$ Value \\
\hline \multicolumn{10}{|c|}{ Apgar score at 5 min (vs 5 min Apgar 9-10) } \\
\hline 5-Apgar 0-6 & & 1.33 & (0.98 to 1.79 ) & & 0.06 & 0.96 & & to 1.35$)$ & 0.80 \\
\hline 5-Apgar 7 & & 1.03 & (0.80 to 1.32$)$ & & 0.85 & 1.10 & & to 1.41$)$ & 0.45 \\
\hline 5-Apgar 8 & & 1.00 & (0.90 to 1.12$)$ & & 0.99 & 0.96 & & to 1.08$)$ & 0.50 \\
\hline Sex of the child, male (vs female) & & 1.97 & (1.83 to 2.11$)$ & & $<0.0001$ & 1.90 & & to 2.04$)$ & $<0.0001$ \\
\hline Child's age at EDI completion (years) & & 0.66 & (0.60 to 0.72$)$ & & $<0.0001$ & 0.84 & & to 0.91$)$ & $<0.0001$ \\
\hline \multicolumn{10}{|l|}{ SES (vs highest SES) } \\
\hline Middle SES & & 2.97 & (2.71 to 3.26 ) & & $<0.0001$ & 2.06 & & to 2.27 ) & $<0.0001$ \\
\hline Lowest SES or income assistance & & 1.62 & (1.50 to 1.75$)$ & & $<0.0001$ & 1.45 & & to 1.56$)$ & $<0.0001$ \\
\hline \multicolumn{10}{|l|}{ Gestational weeks (vs 40 weeks) } \\
\hline 37 & & 1.16 & (1.02 to 1.33$)$ & & 0.03 & 1.18 & & to 1.35$)$ & 0.02 \\
\hline 38 & & 1.04 & (0.94 to 1.15$)$ & & 0.48 & 1.08 & & to 1.20$)$ & 0.16 \\
\hline 39 & & 1.04 & (0.95 to 1.14$)$ & & 0.38 & 1.14 & & to 1.24$)$ & 0.01 \\
\hline 41 & & 0.96 & (0.86 to 1.06$)$ & & 0.40 & 1.00 & & to 1.11$)$ & 0.96 \\
\hline$\geq 42$ & & 1.11 & (0.82 to 1.49$)$ & & 0.51 & 1.19 & & to 1.61$)$ & 0.26 \\
\hline \multicolumn{10}{|l|}{ Birth weight for gestational age (vs AGA) } \\
\hline Large & & 0.99 & (0.90 to 1.09$)$ & & 0.78 & 1.05 & & to 1.16$)$ & 0.37 \\
\hline Small & & 1.26 & (1.12 to 1.42$)$ & & $<0.001$ & 1.51 & & to 1.69$)$ & $<0.0001$ \\
\hline Breastfeeding initiation yes (vs no) & & 1.65 & (1.53 to 1.79$)$ & & $<0.0001$ & 1.63 & & to 1.77$)$ & $<0.0001$ \\
\hline
\end{tabular}

substantial public health significance as a population's Apgar profile could serve as an indicator of the burden of adverse developmental outcomes in children. Additionally, documentation of differences in 5 min Apgar profiles between populations (eg, a lower frequency of infants with a 5 min Apgar score of 9) could lead to an aetiological search for causes and interventions that improve developmental outcomes in children.

The strengths of our study included the ability to access comprehensive health and education-related databases at the population level. The EDI assessment by teachers avoided reliance on parental or self-report of developmental outcomes. Nevertheless, there may have been some individual differences in teachers' ability to evaluate developmental outcomes. ${ }^{19}$ The SES of children was determined by their SES in the first 5 years after birth, although SES at birth would have been preferable for a prognostic model. However, SES as constructed in our study is unlikely to have changed substantially during the study period. Further, our study was restricted to the comparatively healthy subset of all term live births, as children with severe chronic illnesses may not have enrolled in kindergarten or may have enrolled in special needs schools. This was not a serious limitation as our objective was to examine the role of the Apgar score as a marker of childhood development in normal children. 
In summary, our study showed that the risk of developmental vulnerability at 5 years of age was inversely associated with the 5 min Apgar score and that this relation extended across the entire range of scores. Thus, even a minor degree of physiological dysfunction soon after birth, reflected in the Apgar score, may indicate a slightly higher risk for developmental vulnerability in later childhood. Although the low sensitivity of the prognostic model based on the 5 min Apgar score limits the clinical utility of this model for early intervention, the Apgar score might well serve as a population-level indicator of developmental risk.

\section{Author affiliations}

${ }^{1}$ School of Population and Public Health, Faculty of Medicine, University of British Columbia, Vancouver, British Columbia, Canada

${ }^{2}$ Department of Obstetrics \& Gynaecology, Faculty of Medicine, University of British Columbia, Vancouver, British Columbia, Canada

${ }^{3}$ Departments of Pediatrics and Psychiatry, School of Medicine, University of California, San Francisco, California, USA

${ }^{4}$ Department of Community Health Sciences, College of Medicine, University of Manitoba, Winnipeg, Manitoba, Canada

${ }^{5}$ School of Public Health, University of California, Berkeley, California, USA ${ }^{6}$ Brain Research Centre and Department of Medicine (Division of Neurology), Faculty of Medicine, University of British Columbia, Vancouver, British Columbia, Canada ${ }^{7}$ Departments of Internal Medicine and Community Health Sciences, College of Medicine, University of Manitoba, Winnipeg, Manitoba, Canada

Acknowledgements Appreciation is extended to the Manitoba Health Policy Centre (MCHP) staff for providing the data under project (HIPC \#2012/2013-07) and specifically to Charles Burchill at MCHP for his continuous support and guidance throughout the linkage and data analyses process.

Contributors NR: conceptualised and designed the study, carried out all the data analysis, drafted the initial manuscript and contributed to the acquisition of data. WTB: conceptualisation and design of the study, advised in the interpretation of data and critically reviewed and revised the manuscript. MB, DJ and HT assisted in interpretation of the data and critically reviewed the manuscript. RAM contributed to the acquisition of the data, interpretation of the data and critically reviewed the manuscript. KSJ supervised the conceptualisation and design of the study, advised in the interpretation of data and critically reviewed and revised the manuscript. All authors approved the final manuscript as submitted.

Funding This work was supported by the Canadian Institute of Health Research (MOP-119393, PI Helen Tremlett). NR is funded by the Multiple Sclerosis Society of Canada (PhD Research Studentships). She has received travel grants from the Canadian Institutes of Health Research (CIHR) and the endMS Research and Training Network/Multiple Sclerosis Society of Canada. WTB is funded by the Canadian Institute for Advanced Research; the BC Leadership Chair in Child Development also supported this work. MB is the recipient of the Manitoba Centre for Health Policy Population-Based Child Health Research award funded by the Government of Manitoba. KSJ is the recipient of a Chair in maternal, fetal and infant health services research from the CIHR. His work is also supported by the Child and Family Research Institute. HT is funded by the Multiple Sclerosis Society of Canada (Don Paty Career Development Award), Michael Smith Foundation for Health Research, and is the Canada Research Chair for Neuroepidemiology and Multiple Sclerosis. She has received research support from the US National Multiple Sclerosis Society, CIHR, and UK MS Trust; speaker honoraria and/or travel expenses to attend conferences from the Consortium of MS Centres, US National MS Society, the University of British Columbia Multiple Sclerosis Research Program, Bayer Pharmaceutical (speaker, 2010, honoraria declined), Teva Pharmaceuticals (speaker 2011), ECTRIMS (2011, 2012, 2013), UK MS Trust (2011), the Chesapeake Health Education Program, US Veterans Affairs (2012, honorarium declined), Novartis (2012), the Consortium of MS Centers (2013) and Biogen (honorarium declined). Unless otherwise stated, all speaker honoraria are either donated to an MS charity or to an unrestricted grant for use by her research group. RAM receives research funding from CIHR, Multiple Sclerosis Society of Canada, Multiple Sclerosis Scientific Research Foundation, National Multiple Sclerosis Society, Rx \& D Health Research Foundation, and has conducted clinical trials funded by Sanofi-Aventis.

Competing interests None declared.

Provenance and peer review Not commissioned; externally peer reviewed.

Open Access This is an Open Access article distributed in accordance with the Creative Commons Attribution Non Commercial (CC BY-NC 4.0) license, which permits others to distribute, remix, adapt, build upon this work non-commercially, and license their derivative works on different terms, provided the original work is properly cited and the use is non-commercial. See: http://creativecommons.org/ licenses/by-nc/4.0/ 


\section{REFERENCES}

1 Apgar V. A proposal for a new method of evaluation of the newborn. Curr Res Anaesth 1953:32:260-7.

2 Moore EA, Harris F, Laurens KR, et al. Birth outcomes and academic achievement in childhood: a population record linkage study. J Early Child Res 2014;12:234-50.

3 Ehrenstein V, Pedersen L, Grijota M, et al. Association of Apgar score at five minutes with long-term neurologic disability and cognitive function in a prevalence study of Danish conscripts. BMC Pregnancy Childbirth 2009;9:14.

4 Moster D, Lie R, Markestad T. Joint association of Apgar scores and early neonatal symptoms with minor disabilities at school age. Arch Dis Child-Fetal Neonatal Ed 2002;86:F16-21.

5 Marschik PB, Einspieler C, Garzarolli B, et al. Events at early development: Are they associated with early word production and neurodevelopmental abilities at the preschool age? Early Hum Dev 2007;83:107-14.

6 Krebs L, Langhoff-Roos J, Thorngren-Jerneck K. Long-term outcome in term breech infants with low Apgar score-a population-based follow-up. Eur J Obstet Gynecol Reprod Biol 2001;100:5-8.

7 Odd DE, Rasmussen F, Gunnell D, et al. A cohort study of low Apgar scores and cognitive outcomes. Arch Dis Child-Fetal Neonatal Ed 2008;93:F115-20.

8 Stuart A, Olausson PO, Källen K. Apgar scores at 5 minutes after birth in relation to school performance at 16 years of age. Obstet Gynecol 2011;118(2, Part 1):201-8.

9 Health Information Management Branch, Population Report. In: Living MHaH, ed. Winnipeg, Manitoba, 2008.

10 Janus M, Offord DR. Development and psychometric properties of the early development instrument (EDI): a measure of children's school readiness. Can J Behav Sci 2007;39:1-22.

11 Roos LL, Nicol JP. A research registry: uses, development, and accuracy. J Clin Epidemiol 1999;52:39-47.

12 Jutte DP, Roos LL, Brownell MD. Administrative record linkage as a tool for public health research. Annu Rev Public Health 2011;32:91-108.

13 Kramer MS, Platt RW, Wen SW, et al. A new and improved populationbased Canadian reference for birth weight for gestational age. Pediatrics 2001;108:e35.

14 Martens P. Health inequities in Manitoba: is the socioeconomic gap in health widening or narrowing over time? Manitoba Centre for Health Policy, 2010. http://mchp-appserv.cpe.umanitoba.ca/reference/Health_Ineq_final_WEB.pdf

15 Mustard C, Derksen S, Berthelot J, et al. Assessing ecological proxies for household income: a comparison of household income and neighborhood level income measures in the study of population health status. Health Place 1999;5:157-71.
16 Krieger N. Overcoming the absence of socioeconomic data in medical records: validation and application of a census-based methodology. Am J Public Health 1992;82:703-10.

17 Duncan GJ, Dowsett CJ, Claessens A, et al. School readiness and later achievement. Dev Psychol 2007;43:1428.

18 Janus M, Duku E. The school entry gap: socioeconomic, family, and health factors associated with children's school readiness to learn. Early Educ 2007;18:375-403.

19 Measuring in support of early childhood development: The Normative II report. Secondary Measuring in support of early childhood development: The Normative II report [Report]. 2012. http://www.offordcentre.com/readiness/files/updated_ normative_II.pdf

20 Santos R, Brownell MD, Ekuma O, et al. The Early Development Instrument (EDI) in Manitoba: Linking Socioeconomic Adversity and Biological Vulnerability at Birth to Children's Outcomes at Age 5. Winniepg, MB: Manitoba Centre for Health Policy, May, 2012.

21 Are our children ready for school? Manitoba: Healthy Child Manitoba, 2011. http://www.gov.mb.ca/healthychild/edi/edireport_MB_201011.pdf, 2011.

22 Steyerberg EW, Vickers AJ, Cook NR, et al. Assessing the performance of prediction models: a framework for some traditional and novel measures. Epidemiology 2010;21:128-38

23 Janes H, Pepe MS, Gu W. Assessing the value of risk predictions by using risk stratification tables. Ann Intern Med 2008;149:751-60.

24 Adler NE, Boyce T, Chesney MA, et al. Socioeconomic status and health: The challenge of the gradient. Am Psychol 1994;49:15-24

25 Harrison NA, Cooper E, Voon V, et al. Central autonomic network mediates cardiovascular responses to acute inflammation: Relevance to increased cardiovascular risk in depression? Brain Behav Immun 2013;31:189-96.

26 El-Sheikh M, Erath SA, Bagley EJ. Parasympathetic nervous system activity and children's sleep. J Sleep Res 2013;22:282-8.

27 Rees CA. Lost among the trees? The autonomic nervous system and paediatrics. Arch Dis Child 2014;99:552-62.

28 Alkon A, Boyce WT, Tran L, et al. Prenatal adversities and Latino children's autonomic nervous system reactivity trajectories from 6 months to 5 years of age. PLOS ONE 2014;9:e86283.

29 Seidman DS, Laor A, Gale R, et al. Apgar scores and cognitive performance at 17 years of age. Obstet Gynecol 1991;77:875-8.

30 Nelson KB, Ellenberg JH. Apgar scores as predictors of chronic neurologic disability. Pediatrics 1981;68:36-44.

31 Jutte DP, Brownell M, Roos NP, et al. Rethinking what is important: Biologic versus social predictors of childhood health and educational outcomes. Epidemiology 2010;21:314-23. 